PAPER

\title{
Incidence and mutation rates of Huntington's disease in Spain: experience of 9 years of direct genetic testing
}

\author{
M A Ramos-Arroyo, S Moreno, A Valiente
}

J Neurol Neurosurg Psychiatry 2005;76:337-342. doi: 10.1136/jnnp.2004.036806

See end of article for authors' affiliations ....................

Correspondence to: Dr M A Ramos-Arroyo, Servicio de Genética Médica, Hospital Virgen del Camino, C/ Irunlarrea 4, 31008 Pamplona, Navarra, Spain; ma.ramos.arroyo@ cfnavarra.es

Received 18 January 2004 In revised form 2 July 2004 Accepted 30 July 2004
Background: Prior to the discovery of the Huntington's disease (HD) mutation, the prevalence, incidence, and new mutation rates for this disease were based on the presence of progressive choreic movements and a positive family history.

Objective: To evaluate the uptake of the HD genetic analysis in Spain, and to provide additional information on the epidemiology of this disease from the experience of 9 years of direct genetic testing. Methods: From 1994 to 2002, CAG repeat length was determined in 317 patients with symptoms compatible with HD. In all cases, demographic, clinical, and family data were carefully reviewed.

Results: HD diagnosis (CAG repeat length $\geqslant 36$ ) was confirmed in $166(52 \%)$ symptomatic cases. Of these, $76(45.8 \%)$ reported a positive family history and in 21 cases (12.7\%) family history was negative. New mutation events were genetically proven in three families and highly suspected in another, estimating that the minimum new mutation rate for $\mathrm{HD}$ in our population is $>4 \%$, with a potential mutation rate of $8 \%$. More than $16 \%$ of all HD cases had late onset ( $>59$ years) of symptoms, and in three quarters of these the family history was negative. The incidence rate for the autonomous communities of Navarra and the Basque country, based on the number of newly diagnosed cases by genetic testing, was 4.7 per million per year.

Conclusions: Direct HD genetic testing shows that the incidence and mutation rates of the disease are 2-3 times higher than previously reported. We also demonstrated the relevance of CAG repeat length assessment in diagnosing patients with late onset of symptoms and negative family history for HD.
$\mathrm{H}$ untington's disease (HD) has been long considered one of the most serious genetic conditions of adult life. It usually begins with subtle motor anomalies and/or cognitive deficits, followed by progressive chorea, psychiatric disturbances, and mental deterioration. ${ }^{1}$ Prior to the discovery of the HD mutation, diagnosis of HD was based on the presence of progressive choreic movements and cognitive decline, and a positive family history. Prevalence figures, based on these criteria, varied between 4 and 8 per 100000 for different populations. ${ }^{23} \mathrm{~A}$ few studies have analysed the incidence of $\mathrm{HD}$, estimating an average of 2-4 newly symptomatic HD cases per million per year. ${ }^{4}$ To our knowledge, three studies have surveyed the prevalence of the disease in different regions of Spain and no data are available on the incidence rate. While Calcedo-Ordoñez et al ${ }^{5}$ reported in 1970 a prevalence rate of 1.4 per 100000 (Cádiz), two other authors ${ }^{67}$ observed an estimated figure similar to that reported for most European populations (5.4 and 8.4 per 100000 in the regions of Valencia and Salamanca, respectively).

In past years some authors have shown that an important proportion of cases without a positive family history and/or atypical HD neurological symptoms do in fact have a CAG repeat number $\geqslant 36,{ }^{89}$ suggesting that the incidence and mutation rates for this disease are higher than previously reported. Direct mutation analysis for symptomatic and presymptomatic HD testing has been available as a clinical service in the Hospital Virgen del Camino, Pamplona, Spain since August 1993. Our medical genetics laboratory provides HD molecular testing for several regions of Spain. It is the reference HD testing centre for the total population of Navarra and for approximately $75 \%$ of the Autonomous Community of the Basque country. HD genetic testing for the remaining $25 \%$ of the population in this geographic area is performed in the Department of Medical Genetics of the Fundación Jimenez Díaz, Madrid.

The aim of the retrospective study we present was twofold: (a) to evaluate the uptake of the HD genetic test in Spain, and (b) to assess the value of direct CAG repeat analysis as a tool to provide additional information on the epidemiology and natural history of the disease.

\section{PATIENTS AND METHODS}

The study group comprised a total of 317 symptomatic individuals referred to the Medical Genetics Laboratory of the Hospital Virgen del Camino, Pamplona, Spain, for diagnostic testing for HD between October 1993 and December 2002. Direct mutation testing for HD requires the presence of neurological and/or psychiatric symptoms or signs compatible with this disease, as well as a pedigree with family history information on HD and/or other neurodegenerative disorders. Demographic data including age at onset, age at referral, and place of residence are also recorded.

A family history was considered to be positive when there was at least one other family member with an established (clinically or genetically) diagnosis of HD. A family history was considered as negative when no other siblings had signs or symptoms of HD, and the parents were either alive and healthy or did not have a neurological/psychiatric disorder. When one or more relatives had neurological and/or psychiatric symptoms compatible with $\mathrm{HD}$, the family history was considered as suspected. If no information could be obtained, the family history was considered as unknown.

Determination of the CAG repeats was performed in DNA samples isolated from venous blood, using PCR with primers that flank the CAG repeat, as previously described. ${ }^{10}{ }^{11}$ CAG

Abbreviations: HD, Huntington's disease 
repeat number $\geqslant 36$ was considered as expanded and thus diagnostic of HD. More detailed clinical and pedigree information was obtained in cases with discrepancy between the family history and the CAG repeat number. In families where a new mutation event was suspected, haplotyping was performed with a 13 allele VNTR at D4S95, the CAG and the CCG repeats in the HD gene. When parents were available, paternity testing was carried out by molecular analysis of VNTRs at loci D1S7, D7S21, and D12S11.

The incidence of HD for the communities of the Basque Country and Navarra was calculated on the basis of the number of clinically symptomatic cases newly diagnosed by genetic testing per million inhabitants per year during the period 1994-2002. The HVC Medical Genetics Laboratory is a national reference centre for HD in Spain; it is the only diagnostic genetic testing laboratory for this disease for Navarra and is the major HD reference laboratory for other communities. In order to maximise the possibilities of complete ascertainment of new cases, we also searched for additional patients from the Basque country who might have been referred to other HD diagnostic genetic centres in Spain. In addition, patients who underwent presymptomatic testing and became symptomatic within the study period were also included. Annual population estimates for the Basque country and Navarra were obtained through the Instituto Nacional de Estadística.

\section{Statistical analyses}

Differences between groups according to sex, molecular testing results, family history, age of onset, and age at referral were analysed by $\chi^{2}$ test. Mean values were compared by Student's $t$ test. Differences in the number of referrals and diagnostic testing results over time were assessed by linear regression analysis. Data were analysed using the Statistical Package for Social Sciences (SPSS) program, and p <0.05 was considered statistically significant.

\section{RESULTS}

\section{Demographics}

A total of 317 symptomatic individuals (145 males and 172 females) were referred for HD diagnostic testing: 45 from Navarra, 133 from the Basque Country, 64 from the Baleares Islands, 31 from Aragón, and 44 from other regions of Spain. Of these, $166(52.4 \%)$ (77 (46.4\%) men and 89 (53.6\%) women) had a CAG repeat number $\geqslant 36$ (table 1 ). The remaining 151 of the $316(47.6 \%)$ individuals were HD negative (CAG repeat number $<36$ ) and the male to female ratio was $1: 1.2$. The number of referrals and the proportion of non-affected versus affected individuals showed a significant $(p=0.01)$ increasing trend over time (fig 1$)$, but the total number of positive cases/year did not vary significantly (mean (SD) 16.4 (3.5)).

Data on age of onset were obtained in 171 individuals of the total cohort. The mean (SD) age of onset was significantly

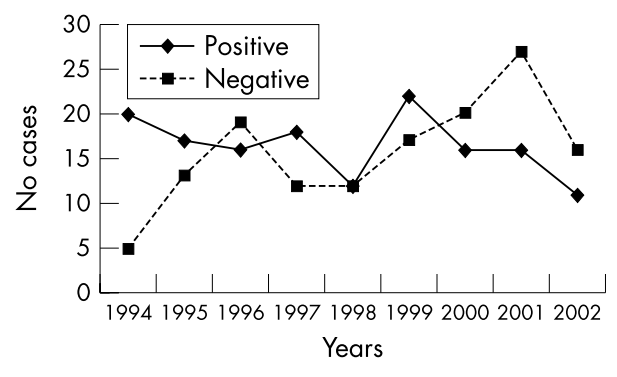

Figure 1 Number of positive and negative genetic test results for Huntington's disease per year (1994-2002).
Table 1 Demographics and genetic test results for Huntington's disease

\begin{tabular}{|c|c|c|c|}
\hline & $\begin{array}{l}\geqslant 36 \\
\text { repeats }\end{array}$ & $\begin{array}{l}<36 \\
\text { repeats }\end{array}$ & Total \\
\hline \multicolumn{4}{|l|}{ Referrals } \\
\hline Navarra & $23(51.1)$ & $22(48.9)$ & $45(100)$ \\
\hline Basque country & $72(54.1)$ & $61(45.9)$ & $133(100)$ \\
\hline Baleares & $25(39.1)$ & $39(60.9)$ & $64(100)$ \\
\hline Aragón & $21(67.7)$ & 10 (32.3) & $31(100)$ \\
\hline Others & $25(56.8)$ & 19 (43.2) & $44(100)$ \\
\hline Total & $166(52.4)$ & $151(47.6)$ & $317(100)$ \\
\hline \multicolumn{4}{|l|}{ Sex } \\
\hline Males & $77(53.1)$ & $68(46.9)$ & $145(100)$ \\
\hline Females & $89(51.7)$ & 83 (48.3) & $172(100)$ \\
\hline \multicolumn{4}{|l|}{ Age at onset (years) } \\
\hline $0-19$ & $7(6.3)$ & $5(8.5)$ & \\
\hline $20-39$ & $38(33.9)$ & $6(10.2)$ & \\
\hline $40-59$ & $49(43.7)$ & 19 (32.2) & \\
\hline$>59$ & $18(16.1)$ & $29(49.1)$ & \\
\hline Total & 112 & 59 & \\
\hline \multicolumn{4}{|c|}{ Age at referral (years) } \\
\hline $0-19$ & $3(2.1)$ & $5(3.6)$ & \\
\hline $20-39$ & $28(18.9)$ & $15(10.9)$ & \\
\hline $40-59$ & 61 (41.2) & $41(29.7)$ & \\
\hline$>59$ & $56(37.8)$ & $77(55.8)$ & \\
\hline Total & 148 & 138 & \\
\hline
\end{tabular}

lower $(\mathrm{p}<0.001)$ among HD positive cases $(43.7(15.0))$ than in the group of individuals with a CAG repeat number $<36$ (53.4 (18.1)). More than $16 \%$ of cases that tested positive for HD and $49.1 \%$ of those that tested negative had a late onset of symptoms ( $>59$ years of age). Among these late onset cases, the number of women was higher than the number of men, with a male to female ratio of $1: 2$ for patients with CAG $\geqslant 36$ and 1:3.1 among those with CAG $<36$. The mean age at diagnosis also differed significantly between HD positive and HD negative groups (52.5 (15.2) v 57.7 (17.7); $\mathrm{p}=0.01)$. A total of $56(37.8 \%)$ patients with CAG $\geqslant 36$, and $77(55.8 \%)$ of those with HD-like symptoms, were $>59$ years of age. Women were over-represented in both groups, with a male to female ratio of $1: 1.4$ and $1: 1.8$, respectively.

The number of CAG repeats in the expanded allele ranged from 38 to 70 with a mean size of $46.3(6.0)$ repeats. There was a significant inverse correlation $(\mathrm{p}=0.002)$ between the number of CAG repeats and age of onset. Cases with juvenile HD had expansions > 7 CAG repeats, while a greater range was observed among cases with adult onset. The number of CAG repeats was significantly different $(\mathrm{p}=0.004)$ between patients with maternal $(44.7(4.0))$ and paternal inheritance (49.8 (8.7)).

\section{Incidence}

The incidence of HD was calculated for Navarra and the Basque country. A total of 111 individuals with a repeat CAG number $\geqslant 36$ became symptomatic between 1994 and 2002. Of these, 85 were tested in the HVC Medical Genetic Laboratory and 22 others had been referred and tested positive at the Fundación Jimenez Diaz in Madrid. The remaining four HD positive patients underwent presymptomatic testing and became symptomatic during the study period. The overall mean minimum incidence was 4.7 per million per year: 4.6 per million in Navarra and 4.8 per million in the Basque country.

\section{Family history}

Table 2 shows the distribution of cases by genetic test results, sex, and family history. More than $90 \%$ of cases with an established HD diagnosis in their families and 59\% of those with suspected family history had a CAG expansion. Among 
Table 2 Distribution of cases by genetic results, sex and family history

\begin{tabular}{|c|c|c|c|c|c|c|c|c|c|}
\hline \multirow{2}{*}{$\begin{array}{l}\text { Family } \\
\text { history }\end{array}$} & \multicolumn{3}{|c|}{$\geqslant 36$ repeats } & \multicolumn{3}{|c|}{$<36$ repeats } & \multicolumn{3}{|l|}{ Total } \\
\hline & Males & Females & Total & Males & Females & Total & Males & Females & Total \\
\hline Positive & $38(49.3)$ & $38(42.7)$ & $76(45.8)$ & $3(4.4)$ & $5(6.0)$ & $8(5.3)$ & $41(28.3)$ & $43(25.0)$ & $84(26.5)$ \\
\hline Suspected & $27(35.1)$ & 32 (36.0) & 59 (35.5) & $20(29.4)$ & $21(25.3)$ & $41(27.2)$ & 47 (32.4) & $53(30.8)$ & $100(31.5)$ \\
\hline Negative & $7(9.1)$ & 14 (15.7) & 21 (12.7) & 37 (54.4) & $50(60.3)$ & 87 (57.6) & $44(30.3)$ & 64 (37.2) & 108 (34.1) \\
\hline Unknown & $5(6.5)$ & $5(5.6)$ & $10(6.0)$ & $8(11.8)$ & $7(8.4)$ & $15(9.9)$ & $13(9.0)$ & $12(7.0)$ & 25 (7.9) \\
\hline Total & 77 (100) & $89(100)$ & $166(100)$ & $68(100)$ & $83(100)$ & 151 (100) & $145(100)$ & $172(100)$ & $317(100)$ \\
\hline
\end{tabular}

cases with negative family history, this figure was $19.4 \%$. No significant differences by gender were observed in any of the groups.

\section{Family history in the group of patients with CAG repeat number $\geqslant 36$}

Less than $50 \%$ of all HD cases had an established family history, and in $12.7 \%$ additional patients, complete absence of family history for neuropsychological disorders was reported.

The mean age of onset and mean age at referral were significantly higher among EH patients with negative family history than in those with an established or suspected HD family history (age of onset 52.3 (16.1) $v 41.6$ (14.2); $\mathrm{p}=0.01 ;$ age at referral 62.3 (15.4) $v$ 50.5 (14.5); $\mathrm{p}=0.002$ ). Interestingly, $30 \%$ of $\mathrm{HD}$ cases with negative family history had a late onset of symptoms and 55\% were $>59$ years of age at the time of referral. These proportions are significantly higher than those observed among HD patients with positive or suspected family history $(12.4 \%$ late onset cases; $28.4 \%$ with age at referral $>59$ years).

The clinical and family history details of the 21 HD patients with negative family history were carefully reviewed. In 10 cases, one or both parents had died before the age of 65 years, so that normal inheritance of HD through the deceased parent could not be ruled out. In one additional case, both parents had died in their 70s of unknown causes, but two uncles committed suicide late in life (>60 years of age), which might indicate an underlining neuropsychological disorder, including HD. For the remaining 10 cases, no signs or symptoms of neurodegenerative disorders were recorded, and parents were either alive or had died without symptoms compatible with HD at the age of 65 years or older (table 3 ). Six of these families were considered possible new mutations as additional DNA molecular analysis could not be carried out in their relatives. In the other four cases, a true new mutation event was highly suspected by the identification of an intermediate allele (30-38 CAG repeats) in one or more asymptomatic first degree relatives. Both parents were available in two families and non-paternity was excluded by molecular analysis. Further haplotype analysis showed that, in all four cases, intermediate alleles occurred on the HD chromosome of the family (fig 2). Expansion of an intermediate allele, paternally inherited, was demonstrated in three families, confirming a new mutation event.

\section{Family history in the group of patients with CAG repeat number $<36$}

More than $57 \%(\mathrm{n}=87)$ of the 151 individuals with a CAG repeat number $<36$ had a negative family history, and in 15 other cases $(9.9 \%)$ the family history was unknown (table 2 ). Forty one $(27.2 \%)$ patients reported having one or more relatives with neuropsychiatric symptoms compatible with $\mathrm{HD}$, and in eight $(5.3 \%)$ additional instances, the diagnosis of
HD had been previously established in one or more family members.

The clinical and family history details of the eight patients with HD-like symptoms and positive family history for this disease are summarised in table 4 . For cases A to C, a CAG expansion was documented in one or more family members. Patient A was referred for HD molecular testing at the age of 44 years, presenting with jerky movements of the neck and tremor of the hands. Two years earlier he had been diagnosed with bipolar disorder. A CAG expansion was demonstrated in his mother and aunt. Patient B was a 63 year old female who had a long term depressive disorder associated with involuntary movements, parkinsonian type, of the head and lower extremities, as well as progressive memory loss. A brother and a sister had a CAG repeat number $\geqslant 36$. Case $C$ was 67 years of age at referral, presenting with walking difficulties and tremor of the right hand. Symptoms had been slowly progressive over the previous 10 years. A brother and a sister showed similar clinical signs. Two other siblings had clear progressive chorea and dementia, and the diagnosis of HD was confirmed by DNA molecular testing. Two additional cases (D and E) with HD-like symptoms and a CAG in the normal range had a second degree relative clinically diagnosed with HD.

Patients $\mathrm{F}$ to $\mathrm{H}$ were members of the same family. Patients $\mathrm{F}$ and $\mathrm{G}$ were the oldest siblings of a kindred of four. Their ages at referral were 52 and 51 years, respectively. They presented with progressive involuntary movements of the face and upper extremities combined with a depressive disorder since their early 40s. Their mother had died at the age of 68 years and presented similar neuropsychiatric symptoms. A son of patient G showed choreic movements and was referred for molecular testing at the age of 30 years. In all three cases, the number of CAG repeats was $<36$.

\section{DISCUSSION}

Availability of the DNA molecular testing for HD has greatly improved the diagnosis of HD, allowing identification of cases with atypical symptoms and those with absence of family history. ${ }^{12}{ }^{13}$ In our study we found that $>54 \%$ of symptomatic patients with an expanded CAG repeat did not have an informative family history. This proportion is substantially higher than those reported in Leyden ${ }^{13}$ $(38.9 \%)$ and in British Columbia $(29.8 \%)^{14}$, and may either reflect a higher rate of new mutations in our population, or most likely, difficulties in verifying the family history in the absence of a centralised register of HD in Spain.

Precise estimates of the new mutation rate are very difficult to make for a late onset disorder such as HD. Prior to the identification of the HD mutation in 1993, new mutations were thought to be a rare event. ${ }^{2}{ }^{15}$ More recent studies, based on the DNA analysis of sporadic cases, have observed that around 3\% of affected patients may be the result of a CAG expansion of an intermediate allele. ${ }^{16}$ In our study, 10 of the 166 cases with an expanded CAG repeat had 

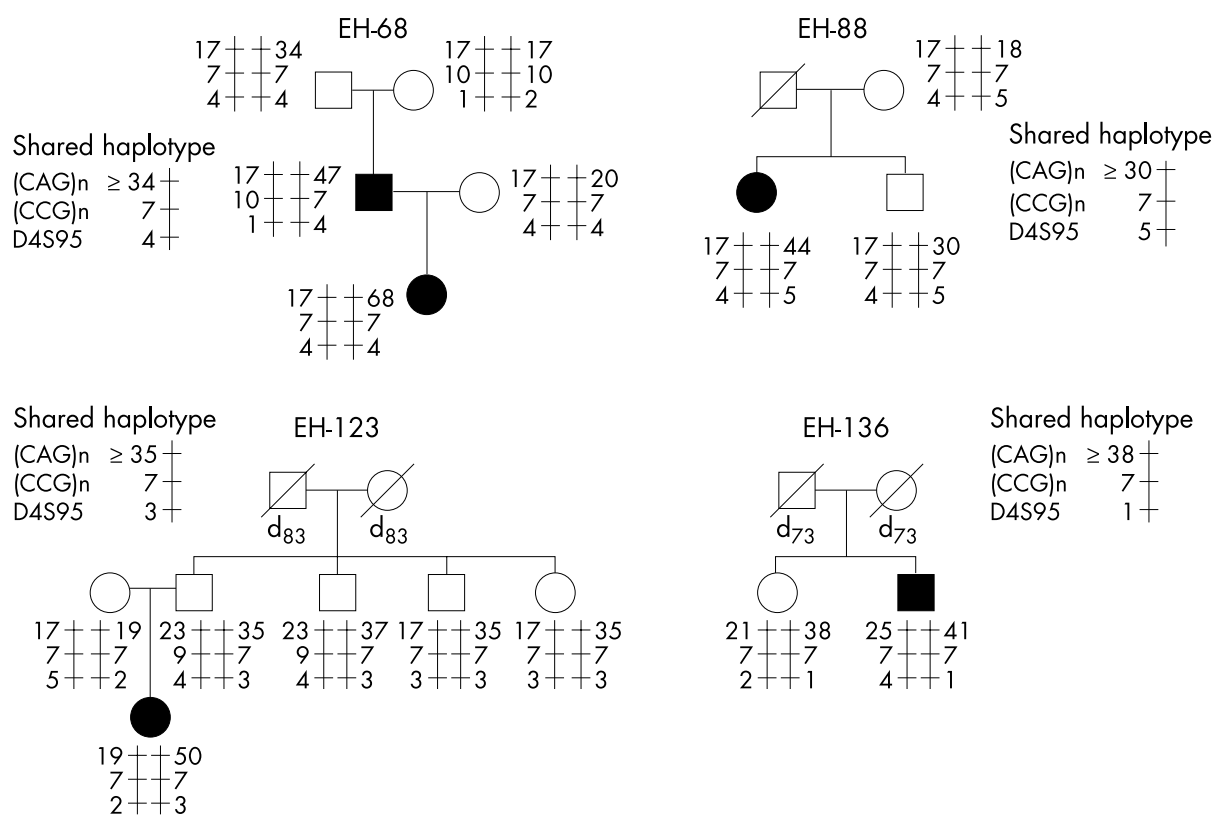

Figure 2 Pedigrees and constructed haplotypes of the four families with an intermediate HD allele (30 to 38 CAG repeats). d, Age of death.

no family history of neuropsychological disorders, and their parents had lived at least until the age of 65 years without symptoms of HD. These families could represent either nonpaternity, nonpenetrance of a CAG repeat or a new HD mutation. Non-paternity could be ruled out in all four cases for all practical purposes, as it was excluded in two instances by molecular analysis, and the likelihood of false paternity having a first degree relative carrying a 30-38 CAG repeat allele that shared the HD family haplotype, as demonstrated in the other families, is negligible. While incomplete penetrance of a CAG repeat in the lower affected range (36-39 repeats) cannot be completely ruled out in family EH136, we were able to demonstrate the expansion of an intermediate allele $(30-35)$ in the remaining three families, confirming a de novo mutation event. We therefore conclude that the minimum HD new mutation rate for the Basque country and Navarra is $4.7 \%$, with a potential new mutation rate of $8.1 \%$. Comparable estimates were observed when including data from other regions of Spain $3 \%$ and $7 \%$, respectively), for which access to detailed family data may have been more limited. These findings support the hypothesis of Falush et al, ${ }^{17}$ who, based on the analysis of the pattern of allele flow, estimated that the new mutation rate for HD is likely to be $>10 \%$. Further studies, including genetic testing of asymptomatic family members, will be needed to confirm that the supposed isolated cases are indeed new mutations.

The high rate of new mutations observed in our study may reflect, at least in part, a higher ascertainment of late onset cases using the direct DNA test. Our data show that $16.1 \%$ of cases with a CAG repeat $\geqslant 36$, for whom data were available, had onset at the age of 60 years or older. A similar proportion of late onset HD cases has been reported by Almqwist et al, ${ }^{14}$ but it is 3-4 times higher than the figures previously estimated. ${ }^{18-20}$ It is interesting to note that $72 \%$ of patients with a CAG expansion and late onset of symptoms did not have a previously established HD family history. Furthermore, when we analysed the data on age at referral, we observed that nearly $50 \%$ of all cases with a CAG expansion were $>60$ years of age when they received diagnostic confirmation of the disease, and $75 \%$ of these

\begin{tabular}{|c|c|c|c|c|c|}
\hline $\begin{array}{l}\text { New mutation } \\
\text { family number }\end{array}$ & $\begin{array}{l}\text { Age of } \\
\text { onset } \\
\text { (years) }\end{array}$ & $\begin{array}{l}\text { Age at } \\
\text { referral } \\
\text { (years) }\end{array}$ & $\begin{array}{l}\text { Mother's information } \\
\text { Age at death (cause) }\end{array}$ & $\begin{array}{l}\text { Father's information } \\
\text { Age at death (cause) }\end{array}$ & Other relatives' information \\
\hline \multicolumn{6}{|l|}{ Proven } \\
\hline EH-68 & 30 & 40 & $\begin{array}{l}\text { Alive at } 70 \text { years. } \\
\text { CAG repeat: } 17 / 17\end{array}$ & $\begin{array}{l}\text { Alive at } 75 \text { years. } \\
\text { CAG repeat: } 17 / 34\end{array}$ & Daughter: CAG repeat 17/68 \\
\hline EH-88 & 38 & 44 & $\begin{array}{l}\text { Alive at } 76 \text { years. } \\
\text { CAG repeat: } 17 / 18\end{array}$ & $? ?$ & Brother: CAG repeat $17 / 30$ \\
\hline EH-123 & 29 & 34 & $\begin{array}{l}\text { Alive at } 59 \text { years. } \\
\text { CAG repeat: } 17 / 19\end{array}$ & $\begin{array}{l}\text { Alive at } 59 \text { years. } \\
\text { CAG repeat: } 23 / 35\end{array}$ & $\begin{array}{l}\text { Paternal uncles/aunts: CAG repeat } 17- \\
23 / 35-37\end{array}$ \\
\hline \multicolumn{6}{|l|}{ Highly suspected } \\
\hline EH-136 & $? ?$ & 60 & 73 (cardiac arrest) & 73 (prostate cancer) & Sister: CAG repeat $21 / 38$ \\
\hline \multicolumn{6}{|l|}{ Possible } \\
\hline EH-103 & ?? & 77 & 70 (thrombosis) & 70 (liver disease) & \\
\hline $\mathrm{EH}-150$ & 67 & 77 & 93 (old age) & 87 (old age) & \\
\hline EH-181 & 59 & 69 & 84 (old age) & 67 (tuberculosis) & \\
\hline $\mathrm{EH}-274$ & 59 & 69 & 81 (cardiac arrest) & 83 (prostate cancer) & \\
\hline $\mathrm{EH}-301$ & 52 & 62 & 75 (leukaemia) & 77 (pulmonary disease) & \\
\hline EH-327 & 75 & 78 & 75 (pulmonary disease) & 76 (gastric cancer) & \\
\hline
\end{tabular}


Table 4 Clinical information on cases with positive family history for HD and CAG repeats $<36$

\begin{tabular}{llllllll}
\hline Case & $\begin{array}{l}\text { Family } \\
\text { number }\end{array}$ & $\begin{array}{l}\text { Age at } \\
\text { referral } \\
\text { (years) }\end{array}$ & $\begin{array}{l}\text { Abnormal } \\
\text { movement }\end{array}$ & $\begin{array}{l}\text { Psychiatric } \\
\text { disorder }\end{array}$ & Dementia & Family history & Final diagnosis \\
\hline A & 188 & 44 & + & + & - & HD confirmed mother and aunt & Bipolar disorder. Somatisation \\
B & 255 & 63 & + & + & + & HD confirmed two siblings & Encephalopathy. Parkinson's disease \\
C & 156 & 67 & Tremor & - & - & HD confirmed two siblings & Essential tremor \\
D & 382 & 77 & + & - & - & HD confirmed paternal aunt & Senile chorea \\
E & 399 & 66 & Parkinsonism & - & - & HD diagnosis aunt & Atypical Parkinson's disease \\
F & 92 & 52 & + & + & - & Psychiatric disorder mother & Phenocopy \\
G & 92 & 51 & + & + & - & Brother of F & Phenocopy \\
H & 92 & 30 & + & - & - & Niece of F & Phenocopy \\
\hline
\end{tabular}

did not have a positive family history. These findings have important clinical and social implications. Healthcare providers should be aware that an important proportion of cases do have a late onset of symptoms, and in most cases, families are not aware of being at risk for a severe neuropsychiatric disorder such as HD. Detection of late onset cases would make it possible to inform and counsel other family members, particularly younger generations, who are likely to be more seriously affected by inheriting a CAG repeat amplification.

In concordance with the aforementioned findings for family history and onset of the disease, we observed a high incidence rate of HD in our population. For the period 19942002, we found that the overall annual incidence rate in Navarra and the Basque country was 4.7 per million per year. We could argue that the number of HD positive cases referred for DNA analysis during the first years after the genetic test was available may not reflect the newly diagnosed cases during this period, as clinicians may have used the test to confirm a previously established clinical diagnosis. However, when we recalculated the overall incidence rate for the periods 1995-2002 and 1996-2002, we obtained comparable estimates (4.6 and 4.7 per million per year, respectively) to the complete study period. We therefore conclude that the annual incidence rate in our population is higher than those reported in most populations before the HD genetic test was available. Similar results have been shown for British Columbia, based on the analysis of HD patients newly diagnosed from 1996 to $1999,{ }^{14}$ and as those authors pointed out, these figures should be considered as minimum incidence rates, given that there may well be additional cases with onset during the study period, who did not have access to genetic testing to confirm the clinical diagnosis.

Another interesting finding of our study was the high proportion $(9.2 \%)$ of symptomatic patients with a positive HD family history who had CAG repeats within the normal range. Follow up of these patients and a more detailed clinical investigation showed that the half $(4 / 8)$ were clinical misdiagnoses. One patient suffered from essential tremor, a disorder that also segregated in the family in a autosomal dominant mode. Four additional cases were finally diagnosed as senile chorea, Parkinson's disease, and bipolar disorder. Only three members of the one single family, who presented with HD-like symptoms and did not fulfil the criteria for other neuropsychological illnesses, could specifically be considered as possible phenocopies in our study sample. This would represent $1.8 \%$ of symptomatic cases with positive or suspected family history. These results on misdiagnosis and possible phenocopies are comparable with previous studies, ${ }^{21}$ and reinforce the need for careful clinical investigation of symptomatic patients and the relevance of DNA genetic testing to confirm the diagnosis in HD families.

The data presented in this study reflect the development and experience of direct genetic testing of HD in Spain. The uptake of CAG repeat analysis in our population showed a significantly increasing trend over time. However, while the proportion of test results with a CAG expansion decreased from $69 \%$ during 1994-1995 to 30\% during the last 2 years of the study (2001-2002), the total number of results with CAG $\geqslant 36$ did not change significantly over time. These results are not quite comparable to previous studies. Harper et $a^{22}$ reported similar proportion of abnormal versus normal test results during the period 1995-1997 (39\%), but observed a tendency to increase rather than to decrease over time. In contrast, experience in British Columbia for the years 1993-1999 showed that the number of referrals and a high proportion $(69 \%)$ of the test results with CAG expansion remained stable over the years. ${ }^{14}$ These differences may reflect variation in the clinical criteria for requesting molecular testing, but more likely, show differences in healthcare systems between countries. Our laboratory is a national HD reference diagnostic centre within the public healthcare system in Spain, so that referring hospitals do not get charged for the service. In these circumstances, medical specialists may be more likely to request genetic testing than would those working within a private healthcare system.

Data from our experience in symptomatic testing for HD show that the incidence and mutation rates for HD in our population is twice as high as that previously estimated. The study also highlights the importance of the direct assessment of CAG repeat length in diagnosing patients with atypical HD symptoms, including late age of onset and uninformative family history. Given the genetic implications of this disease, diagnostic confirmation in individuals with an established HD family history is also strongly recommended.

\section{AKNOWLEDGEMENTS}

We thank the collaboration of all patients and their families, and the medical professionals who provided the clinical and family history information This study was supported by a grant of the Departamento de Salud del Gobierno de Navarra.

\section{Authors' affiliations}

M A Ramos-Arroyo, S Moreno, A Valiente, Servicio de Genética Médica, Hospital Virgen del Camino, Pamplona, Spain

Competing interests: none declared

\section{REFERENCES}

1 Harper PS. Huntington's disease. London: WB Saunders, 1996.

2 Harper PS. The epidemiology of Huntington's disease. Hum Genet 1992;89:365-76.

3 Hayden MR. Huntington's chorea. New York: Springer-Verlag, 1981.

4 Kokmen E, Ozekmekci FS, Beard CM, et al. Incidence and prevalence of Huntington's disease in Olmsted County, Minnesota (1950 through 1989). Arch Neurol 1994;51:696-8.

5 Calcedo-Ordoñez A. Enfermedad de Huntington en la provincia de Cádiz. Rev Clin Esp 1970;119:333-44.

6 Ruiz JJ, Ortin A, Cacho J, et al. Corea de Huntington: estudio epidemiológico en la provincia de Salamanca. Arch Neurobiol 1985;48:302-3. 
7 Burguera JA, Solis P, Salazar A. Estimate of the prevalence of Huntington disease in the Valencia region using the capture-recapture method. Rev Neurol 1997; 25:1845-7.

8 MacMillan J, Davies P, Harper PS. Molecular diagnostic analysis for Huntington's disease. A prospective evaluation. J Neurol Neurosurg Psychiatry 1995;58:496-8.

9 Goldberg YP, Kremer B, Andrew SE, et al. Molecular analysis of new mutations for Huntington's disease: intermediate alleles and sex of origin effects. Nature Genet 1993:5:174-9.

10 Huntington's Disease Collaborative Research Group. A novel gene containing a trinucleotide repeat that is expanded and unstable on Huntington disease chromosomes. Cell 1993;72:71-83.

11 Warner JP, Barron LH, Brock DJ. A new polymerase chain reaction (PCR) assay for the trinucleotide repeat that is unstable and expanded on Huntington's disease chromosomes. Mol Cell Probes 1993.7:235-9.

12 Mandich P, Di Maria E, Bellone E, et al. Molecular analysis of the IT1 5 gene in patients with apparently 'sporadic' Huntington's disease. Eur Neurol 1996:36:348-52.

13 Siesling S, Vegter-van de Vlis $M$, Losekoot $M$, et al. Family history and DNA analysis in patients with suspected Huntington's disease. I Neurol Neurosurg Psychiatry 2000 Jul;69:54-9.

14 Almqvist EW, Elterman DS, MacLeod PM, et al. High incidence rate and absent family histories in one quarter of patients newly diagnosed with Huntington disease in British Columbia. Clin Genet 2001;60:198-205.
15 Conneally PM. Huntington disease: genetics and epidemiology. Am J Hum Genet 1984;36:506-26.

16 Andrew SE, Goldberg YP, Hayden MR. Rethinking genotype and phenotype correlations in polyglutamine expansion disorders. Hum Mol Genet 1997;6:2005-10.

17 Falush D, Almqvist EW, Brinkmann RR, et al. Measurement of mutational flow implies both a high new-mutation rate for Huntington disease and substantial underascertainment of late-onset cases. Am J Hum Genet 2001;68:373-85.

18 Andrew SE, Goldberg YP, Kremer B, et al. The relationship between trinucleotide (CAG) repeat length and clinical features of Huntington's disease. Nature Genet 1993;4:398-403.

19 James CM, Houlihan GD, Snell RG, et al. Late-onset Huntington's disease: a clinical and molecular study. Age Ageing 1994;23:445-8.

20 Sanchez A, Mila M, Castellvi-Bel S, et al. Molecular analysis of the IT15 gene in 79 Spanish families with Huntington's disease: diagnostic confirmation and presymptomatic diagnosis. Med Clin (Barc) 1997;108:687-90.

21 Andrew SE, Goldberg YP, Kremer B, et al. Huntington disease without CAG expansion: phenocopies or errors in assignment? Am J Hum Genet 1994:54:852-63.

22 Harper PS, Lim C, Craufurd D, on behalf of the UK Huntington's Disease Prediction Consortium. Ten years of presymptomatic testing for Huntington's disease: the experience of the UK Huntington's Disease Prediction Consortium. J Med Genet 2000;37:567-71.

\section{$\mathrm{ECHO}$}

\section{Neurological concomitants of uveitis \\ J R Smith, J T Rosenbaum}

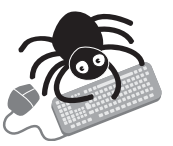

Please visit the Journal of Neurology, Neurosurgery, and Psychiatry website [www. jnnp.com] for a link to the full text of this article.
Aim: To describe the prevalence and types of neurological disease that occur in association with uveitis.

Methods: Retrospective review of medical records of patients attending a tertiary referral uveitis service over a 15 year period.

Results: Of 1450 patients with uveitis, 115 (7.9\%) had neurological disease that was considered to be causally related to the eye inflammation. The most frequent neurological associations were Vogt-Koyanagi-Harada disease, primary central nervous system lymphoma, multiple sclerosis, and herpes virus infections.

Conclusions: Neurological disease is common among patients attending a uveitis service. The distinctive characteristics of the uveal inflammation may be useful in diagnosing the neurological disease.

A British Journal of Ophthalmology 2004;88:1498-1499. 